\title{
Driver Genes of Locally Advanced Well- differentiated Thyroid Cancer: Genetic Landscape Based on the TCGA Database
}

\author{
Ah Ra Jung \\ Eulji University Hospital \\ Dong Hyuk Jang \\ Eulji University Hospital \\ Jung Ho Choi \\ Eulji University Hospital \\ Yoon Se Lee ( $\nabla$ manselee@gmail.com ) \\ Asan Medical Center
}

\section{Research Article}

Keywords: Differentiated thyroid cancer, Locally advanced, Driver genes, TCGA

Posted Date: June 8th, 2021

DOl: https://doi.org/10.21203/rs.3.rs-582794/v1

License: (c) (i) This work is licensed under a Creative Commons Attribution 4.0 International License. Read Full License 


\section{Abstract}

Background: Despite the usually favorable prognosis of well-differentiated thyroid cancer (WDTC) following appropriate treatment, advanced T-staged WDTCs are associated with poor prognosis. This study focused on identifying the driver genes of locally advanced WDTC by analyzing the TCGA cohort.

Methods: We analyzed data on 501 patients with WDTC from the TCGA cohort. Patients were classified into two subgroups of pathological T4 stage or T1-3 stage (Cluster1 and Cluster2, respectively). The mRNA expression differences between subgroups were compared for several genes in the TCGA cohorts.

Results: Cluster1 included 23 patients with pathological T4 classification (Papillary=21/Follicular=2) and Cluster2 included 478 patients (Papillary=371/Follicular=100/Others=7). The Cluster1 subgroup showed worse overall survival than the Cluster2 subgroup $(p<0.05)$. The two subgroups were analyzed for 34 genes reported in previous studies. Known genetic thyroid cancer alterations, including BRAF, RAS, RET, and $A L K$, were not different in the two subgroups. In Cluster1, MET, SERPINA1, TIMP1, PROS1, FN1, $C D K N 2 A$, and $C D K N 2 B$ were significantly elevated, while $T G, D N A H 9, T F F 3, C R A B P 1, T P O, J A K 2, K I T, K D R$, and NFE2L2 were significantly lower compared with the Cluster2 (all, $\mathrm{p}<0.05$ ). A TERT, EIF1AX, and ATM showed significantly frequent somatic mutations in Cluster1 compared to Cluster2. We also identified seven pathways related to the 16 genetic markers.

Conclusions: Locally advanced WDTC presented 16 genetic alterations compared to less aggressive thyroid cancers. Somatic mutations associated with local invasion transformation were identified. Genetic profiles associated with locally advanced WDTC have prognostic significance, but these findings must be validated to further understand the pathway.

\section{Background}

Resectable well-differentiated thyroid cancers (WDTC), including papillary thyroid cancer (PTC) and follicular thyroid cancer (FTC), have a favorable prognosis after surgical treatment with or without adjuvant radioactive iodine therapy. WDTC treatment results are affected by age, gender, aggressive subtypes, and various molecular markers. $(1,2)$ About $10 \%$ of WDTC are composed of locally advanced and resectable WDTC (T4a) invading surrounding structures.(3) It has a two times higher recurrence rate than lower staged WDTC, and about $30 \%$ of advanced thyroid cancer died within a decade.(4,5) Among patients with distant metastasis, T4-staged tumors increased the risk of distant metastasis and decreased recurrence-free survival rate five times compared to early staged WDTC.(6, 7) Compared to confined diseases, locally advanced WDTC requires adjuvant radioactive iodine therapy (RAI), which reduces the risk of recurrence/disease-specific mortality in high-risk patients.(8) External beam radiation therapy (EBRT) is also used to control local recurrence or residual tumors after surgical resection.(9) Despite these treatment modalities, the five-year disease-specific survival rate of T4-staged WDTC ranges from $67.9-87.6 \%$, dependent on the resection margin status.(10) Targeted therapy-based on molecular biomarker investigation may improve treatment outcomes in locally advanced WDTC. 
Most WDTC are associated with alterations in a limited number of driver genes, including point mutations in the BRAF or RAS genes or rearrangements of RET/PTC or PAX8/PPARY, which activate the receptor tyrosine kinase (RTK)/mitogen-activated protein kinase (MAPK) pathway.(11) Accumulated genetic and epigenetic alterations initiate and progress thyroid cancer. Currently, these genetic alterations have been used as a molecular signature to discriminate the type of cancer. Next-generation sequencing (NGS) has recently revealed various genes involved in carcinogenesis and metastasis in various solid cancers.(12)

A pan-cancer study using The Cancer Genome Atlas (TCGA) study has defined genetic alterations in papillary thyroid cancer (PTC) with a focus on low-to-intermediate risk tumors.(13) Previous studies have shown the potential contribution of TERT and various oncogenes (AKT1/PIK3CA and EIF1AX) in the early progression of WDTC and, in particular, report that loss of CDKN2A may be a strong prognostic factor for patients with advanced WDTC.(14) Although the molecular characteristics of WDTC have been analyzed, the underlying mechanism of its progression to locally advanced and resectable WDTC has not been fully elucidated. To overcome the limitations of current treatments for advanced thyroid cancer, the genetic landscape suggests molecular tools for diagnosis and treatment. In this study, we focused on analyzing the TCGA cohort to identify the driver genes of locally advanced WDTC and we evaluated the associations between genetic markers and the prognosis of patients with advanced WDTC.

\section{Methods}

\section{Data sources}

For this study, clinical and genomic data were collected from TCGA thyroid carcinoma. These data can be downloaded from the cBioPortal Browser (https://cbioportal.org/). Clinical information included age, sex, tumor site, TNM staging, recurrence, and survival outcomes. We generated gene expression data for the TCGA cohort by Illumina HiSeq2000 RNA sequencing Version 2 Analysis and transformed and mapped log2-via RSEM.(15) In the cBioPortal thyroid study, there were a total of four cohorts, three of which were WDTC cohorts. The first large-scale integrative genomic analysis of WDTC took place in 2014 (TCGA, Cell 2014). We selected the cohort with the most T4 stage patients (TCGA, Firehose Legacy). Finally, the selected cohort $(n=501)$ included 392 cases of papillary thyroid cancer, 102 cases of follicular thyroid cancer, and seven others. We divided enrolled patients into Cluster1 (T4) and Cluster2 (T1, T2, and T3) according to pathological T classification (Fig. 1).

\section{Pathway analysis}

We used the functional annotation tools from the Database for Annotation, Visualization, and Integrated Discovery (DAVID) bioinformatics resources 6.8.(16) We used the default setting from the tool to map the 16 gene lists to the reference set of direct and indirect relationships, which is a differentially expressed gene list between the two subgroups. P-values were adjusted for multiple testing using the BenjaminiHochberg procedure within the dataset and the Kyoto Encyclopedia of Genes and Genomes (KEGG) pathway module. Significant results were constrained at the 0.05 level after adjustment for multiple testing corrections. 


\section{Statistical analysis}

To test the prognostic significance, only gene expression information with available survival data were used. Overall survival (OS) was defined as the day of surgery to death due to any cause or the last visit. The disease-free survival (DFS) was defined as the day of surgery to the first recurrence at any site or the last visit. Prognostic significance between the two subgroups was estimated by the Kaplan-Meier method.

Fisher's exact test was used to assess the difference in frequency of somatic mutations. P-values of less than 0.05 were considered statistically significant and all statistical tests were two-tailed. Statistical analyses were performed using the IBM ${ }^{\circledR}$ SPSS ${ }^{\circledR}$ Statistics version 24.0 for Windows (IBM Corp., Armonk, NY) and R package version 3.4.4 (http://www.r-project.org).

\section{Results}

\section{Cohort characteristics}

The 501 patients included 135 (26.9\%) males and 366 (73.1\%) females with a median age of 46 years (range, 15-89 years). Cohort characteristics are summarized in Table 1. The most common tumor site was the right lobe $(n=214,42.7 \%)$, followed by the left lobe $(n=173,34.5 \%)$, bilateral $(n=86,17.2 \%)$, isthmus ( $n=22,4.4 \%)$, and others $(n=6,1.2 \%)$. Advanced T classification was found in 192 patients (38.3\%), nodal positivity in 225 patients (44.9\%), and an overall III-IV stage in 165 patients (33.0\%). The most common histological finding was the papillary $(n=392,78.2 \%)$, followed by the follicular $(n=102$, $20.4 \%)$, and others $(n=7,1.4 \%)$. 
Table 1

Patient characteristics $(N=501)$

\begin{tabular}{|ll|}
\hline Variable & N (\%) \\
\hline Gender & \\
\hline Male & $135(26.9)$ \\
\hline Female & $366(73.1)$ \\
\hline Age (years), median (IQR) & $46(35-58)$ \\
\hline Laterality & \\
\hline Right lobe & $214(42.7)$ \\
\hline Left lobe & $173(34.5)$ \\
\hline Bilateral & $86(17.2)$ \\
\hline Isthmus & $22(4.4)$ \\
\hline Others & $6(1.2)$ \\
\hline pT classification & \\
\hline T1 & $142(28.3)$ \\
\hline T2 & $167(33.4)$ \\
\hline T3 & $169(33.7)$ \\
\hline T4 & $23(4.6)$ \\
\hline pN classification & $55(11.0)$ \\
\hline N0 & $226(45.1)$ \\
\hline N1a & $150(29.9)$ \\
\hline N1b & $50(10.0)$ \\
\hline NX & \\
\hline Overall pTNM stage & \\
\hline I & \\
\hline II & \\
\hline III & \\
\hline Papillary & \\
\hline
\end{tabular}

Page 5/17 


\begin{tabular}{|ll|}
\hline Variable & $\mathbf{N}(\%)$ \\
\hline Follicular & $102(20.4)$ \\
\hline Others & $7(1.4)$ \\
\hline
\end{tabular}

\section{Survival outcome of Cluster1 and Cluster2}

Cluster 1 and Cluster 2 consisted of 23 patients (papillary $=21 /$ follicular $=2$ ) and 478 patients (papillary = 371 / follicular $=100 /$ others $=7$ ), respectively. The Kaplan-Meier plots showed that the overall survival (OS) of Cluster1 was significantly worse than Cluster2 ( $p<0.05$, Fig. $2 A)$. The disease-free survival (DFS) of patients in Cluster1 was worse than that of patients in the Cluster2 subgroup ( $p=0.12$, Fig. 2B).

\section{Discover of sixteen driver genes in locally advanced well- differentiated thyroid cancer}

This study analyzed the expression of a variety of genes associated with thyroid cancer, including $B R A F^{V 600 E}, R A S, R E T$, and $A L K$, between two clusters. Griffith et al.(Griffith, et al. 2006) identified 12 important diagnostic biomarkers through a meta-review of gene expression in thyroid cancer. Pozdeyev et al.(17) proposed an updated model of thyroid cancer genetic evolution. We analyzed these genetic alterations.

In Cluster1, expression of MET, SERPINA1, TIMP1, PROS1, FN1, CDKN2A, and CDKN2B were significantly elevated (all, $\mathrm{p}<0.05$, Fig. 3A), while expression of TG, DNAH9, TFF3, CRABP1, TPO, JAK2, KIT, KDR, and NFE2L2 were significantly lower compared with Cluster2 (all, $p<0.05$, Fig. 3B).

\section{Relationship between clusters and somatic mutation}

To investigate somatic mutations between the two clusters in WDTC, we analyzed the somatic mutation data of patients in the TCGA cohort $(n=501)$. We evaluated genes reportedly altered more frequently in advanced differentiated and anaplastic thyroid cancer than in differentiated thyroid cancer. $(18,19)$ (Table S1) The signaling pathways and gene groups seen more frequently in advanced differentiated thyroid cancers are tumor suppressors, the cell-cycle pathway, and the PI3K/AKT pathway.(17) ) Among these genes, A TERT, EIF1AX, and ATM showed a significantly higher frequency of somatic mutation in Cluster1 than in Cluster2 (Fig. 4 and Table S1). There was no difference in the frequency of somatic mutations between the two subgroups for BRAF $V 600 E$ and NRAS.

\section{Pathway analysis}

The genes analyzed with the DAVID functional annotation tools identified 7 significant KEGG pathways based on mutational enrichment (Table 2): proteogloycans in cancer $(p=5.3 e-02)$, the Rap1 signaling pathway $(p=5.8 \mathrm{e}-02)$, and autoimmune thyroid disease $(p=9.4 e-2)$. Moreover, several pathways important for locally advanced cancer were identified, including the PI3K-AKT signaling pathway $(p=$ 
3.1e-3), the pathway in cancer $(p=5.0 e-3)$, focal adhesions $(p=5.6 e-2)$, and the Ras signaling pathway ( $=6.6 \mathrm{e}-2)$.

Table 2

The significant KEGG pathways.

\begin{tabular}{|lllll|}
\hline KEGG pathway & Count & Gene & p-value & FDR \\
\hline PI3K-Akt signaling pathway & 5 & JAK2, KIT, MET, FN1, KDR & $3.1 \mathrm{E}-03$ & $1.6 \mathrm{E}-01$ \\
\hline Pathways in cancer & 5 & $K I T, M E T, C D K N 2 A, C D K N 2 B, F N 1$ & $5.0 \mathrm{E}-03$ & $1.3 \mathrm{E}-01$ \\
\hline Proteoglycans in cancer & 3 & $M E T, F N 1, K D R$ & $5.3 \mathrm{E}-02$ & $6.3 \mathrm{E}-01$ \\
\hline Focal adhesion & 3 & $M E T, F N 1, K D R$ & $5.6 \mathrm{E}-02$ & $5.5 \mathrm{E}-01$ \\
\hline Rap1 signaling pathway & 3 & $K I T, M E T, K D R$ & $5.8 \mathrm{E}-02$ & $4.8 \mathrm{E}-01$ \\
\hline Ras signaling pathway & 3 & $K I T, M E T, K D R$ & $6.6 \mathrm{E}-02$ & $4.7 \mathrm{E}-01$ \\
\hline Autoimmune thyroid disease & 2 & $T G, T P O$ & $9.4 \mathrm{E}-02$ & $5.4 \mathrm{E}-01$ \\
\hline
\end{tabular}

\section{Discussion}

In this study, we demonstrated that the genetic profiles of T4 staged WDTC has genetic alterations, somatic mutations, and a relevant pathway compared to the less aggressive WDTC by analyzing a TCGA cohort. T4 staged WDTC presented 16 genetic alterations compared to less aggressive thyroid cancers, and we identified somatic mutations associated with locally invasion transformations. We also identified seven pathways related to 16 genetic markers. Moreover, we enhanced the current knowledge about the genetic characteristics of locally advanced and resectable WDTC.

WDTC, such as follicular and papillary carcinomas, account for $95 \%$ of all thyroid cancer cases and are generally have a survival rate of almost $100 \%$ when diagnosed early.(20) Approximately $25 \%$ of patients with WDTC develop locally advanced or metastatic disease and locally advanced WDTC is managed differently with adjuvant therapy and surgical treatment.(21) Post-surgical management includes RAI therapy to reduce these locoregional and distant metastasis. When RAI therapy becomes ineffective against differentiated thyroid cancer (DTC), the five-year survival is $<50 \%$ and 10 -year survival is $<10 \%$. $(21,22)$ Of 80 patients with radioactive iodine-refractory DTC, 38 had somatic mutations $(47.5 \%)$, including BRAF ${ }^{V 600 E}, R A S, T P 53, M E T, P I K 3 C A, G N A S$, and TPO.(23) The current study identified the incidence of somatic mutation in $B R A F^{V 600 E}$ at $59.8 \%$, which is similar to the TCGA cohort (58.0\%) in 2014 , whereas only $8.4 \%, 3.5 \%$, and $0.7 \%$ of tumors had RAS mutations (NRAS, HRAS, and KRAS, respectively). The first TCGA cohort discovered two molecular subtypes, $B R A F^{V 600 E_{-}}$like and $R A S$ like, in the papillary thyroid carcinoma.(13) Dedifferentiation is likely to play a role in mitigating responses to RAI treatment and is consistent with the BRAF mutation.(24) However, our results showed no difference in the $B R A F$ mRNA expression level and the frequency of somatic mutations between the two clusters. 
In our study, 23 patients with pathological T4 classification had significantly elevated expression of $C D K N 2 A$ and significantly lower JAK2 compared with Cluster2. Expression of $B R A F, R A S, R E T$, and $A L K$ was not different between the two subgroups. CDKN2A and $C D K N 2 B$ are negative cell-cycle regulators, and their loss due to copy number alterations, inactivating mutations, or epigenetic silencing is one of the most frequently encountered genetic events in human cancer.(25) In thyroid cancer, genetic alterations of $C D K N 2 A$ and $C D K N 2 B$ were seen more frequently in anaplastic thyroid cancer compared with PTC, suggesting a potential role in anaplastic transformation.(17) The prognostic significance of JAK2 has been well described for a variety of cancers, but not advanced thyroid cancer. JAK2 plays a critical role in the signaling of prolactin (PRL) hormone, which may be involved in the development of medullary thyroid carcinoma.(26) Peng et al.(27) examined the TCGA database containing 9,315 tumor samples from 31 cancer types to study the relationship between the mRNA expression of JAK2 and PD-L1 expression. They found that high JAK2 expression was associated with high mRNA expression of PD-L1, which is likely a good indicator for immunotherapy response, including anti-PD-1/PD-L1 therapy. Our results of significantly lower JAK2 expression in Cluster1 compared to Cluster2 may contribute to a treatment strategy that can screen patient populations that may benefit from immunotherapy.

A recent study identified 676 genes associated with an increased risk of PTC recurrence in TCGA data. (28) Chien et al. demonstrated that downregulation of the sodium-iodide symporter SLC5A5 (NIS) was the strongest predictor of a decreased recurrence-free survival.(28) Our study and previous studies have shown the associations of $K I T, T F F 3$, and $T G$ low with increased recurrence rates. Most of the recently discovered targeted therapies inhibit the known oncogenic mechanisms in thyroid cancer initiation and progression, such as the MAPK pathway, PI3K/Akt-mTOR pathways, or VEGF. (29) In clinical trials using trametinib with VEGF inhibitors in advanced DTC patients, $33 \%$ of subjects showed a partial response (PR) and 50\% had stable disease.(30) Bible at el. reported significant activity of single-agent VEGF inhibitor in progressive RAI refractory DTC, with an overall confirmed PR rate of $49 \%$.(31)

The present study revealed that mutations of TERT, EIF1AX, and ATM were found more frequently in Cluster1 than Cluster2. The TERT gene locus was amplified in various cancers, including lung, breast, and cervical cancer,(32) and the prevalence of TERT promoter mutations in thyroid cancer was more frequent in poorly-differentiated and anaplastic thyroid cancers.(33) A recent study identified an EIF1AX mutation in a nodule ultimately diagnosed as oncocytic thyroid carcinoma.(34) EIF1AX mutations are predictive of worse survival in WDTC.(35) This suggests that the treatment strategy can be predicted by markers found in advanced WDTC, unlike the mutations that are the major drivers of WDTC.

Table 3 shows the KEGG pathways of our 16 genes. The growing body of evidence demonstrating the involvement of the PI3K-AKT signaling pathway in thyroid carcinogenesis and drug resistance led to the discovery of several agents targeting key members of this cascade.(29) Multi-kinase inhibitors targeting highly expressed tyrosine kinases in thyroid cancer cells demonstrate a promising anti-tumor activity in vitro and in vivo. $(36,37)$ The few targeted therapies that have been proven effective for advanced WDTC, sorafenib, selumetinib, pazopanib, and sunitinib, have promising results. Due to its high affinity to the VEGF receptor, lenvatinib showed high anti-angiogenic effects in the thyroid cancer mouse model.(38) 
This study has some limitations. High post-ablation stimulated thyroglobulin level ( $\geq 1 \mathrm{ng} / \mathrm{mL})$ and macroscopic ETE were reported as negative prognostic factors in T4a staged WDTC.(39) It is difficult to consider these clinical and pathological factors using TCGA data. Specific clinical factors may help delineate the genomic landscape related to aggressive tumor behavior. Another limitation of this study included not controlling for histologic variants or tumor nodals positive of the well that are differentiated for the thyroid cancer studied. Our study aimed at identifying driver genes and somatic mutations instead of developing a validated prognostic factor in locally advanced WDTC. We hypothesized that using pathway analysis would provide a better understanding of the thyroid carcinogenesis involved in T4 stage tumors.

\section{Conclusions}

A refined classification system that more accurately reflects genotypic and phenotypic differences between locally advanced and resectable WDTC (T4a), which invade the surrounding structures, and other WDTCs will lead to more precise medical and surgical treatment. Our study has provided insight into the molecular pathogenesis of T4 beyond its histological classification. Relevant pathways could be candidates for targeted therapy. Further studies are necessary to translate this study into useful clinical applications in the diagnosis and treatment of T4 staged WDTC.

\section{Abbreviations}

WDTC: Well-differentiated Thyroid Cancer; PTC: Papillary Thyroid Cancer; FTC: Follicular Thyroid Cancer; RAI: Radioactive lodine; EBRT: External Beam Radiation Therapy; NGS: Next-generation Sequencing; TCGA: The Cancer Genome Atlas; DAVID: Database for Annotation, Visualization, and Integrated Discovery; KEGG: Kyoto Encyclopedia of Genes and Genomes; OS: Overall Survival; DFS: Disease-free Survival; DTC: Differentiated Thyroid Cancer.

\section{Declarations}

\section{Acknowledgments}

None to be declared.

\section{Authors' contributions}

ARJ and YSL designed of study and drafted the first manuscript. DHJ and JHC were involved in statistical analysis, creating tables and figures, and drafting the manuscript. ARJ and YSL reviewed manuscript drafts and provided critical revisions. All the authors have read the manuscript and have approved this submission.

\section{Funding}


This research was funded by the National Research Foundation of Korea, grant numbers MSIP; 2016R1C1B1014827 and 2019R1H1A1080141 to Y.S.L.

\section{Availability of data and materials}

The collection of data that supports the findings in this study is available from the cBioPortal Browser (https://cbioportal.org/). Data are available from the authors upon reasonable request and with permission of The Cancer Genome Atlas (TCGA) thyroid carcinoma.

\section{Ethics approval and consent to participate}

No applicable.

\section{Consent for publication}

No applicable.

\section{Competing interest}

The authors have declared no conflicts of interest.

\section{References}

1. Jonklaas J, Sarlis NJ, Litofsky D, Ain KB, Bigos ST, Brierley JD, et al. Outcomes of patients with differentiated thyroid carcinoma following initial therapy. Thyroid. 2006;16(12):1229-42.

2. Yang $C Q$, Gardiner L, Wang H, Hueman MT, Chen D. Creating prognostic systems for welldifferentiated thyroid cancer using machine learning. Frontiers in endocrinology. 2019;10:288.

3. Mazzaferri EL, Jhiang SM. Long-term impact of initial surgical and medical therapy on papillary and follicular thyroid cancer. The American journal of medicine. 1994;97(5):418-28.

4. Emerick GT, Duh QY, Siperstein AE, Burrow GN, Clark OH. Diagnosis, treatment, and outcome of follicular thyroid carcinoma. Cancer. 1993;72(11):3287-95.

5. Salvesen H, Njølstad P, Akslen L, Albrektsen G, Søreide O, Varhaug J. Papillary thyroid carcinoma: a multivariate analysis of prognostic factors including an evaluation of the p-TNM staging system. The European journal of surgery= Acta chirurgica. 1992;158(11-12):583-9.

6. Abraham E, Roshan D, Tran B, Wykes J, Campbell P, Ebrahimi A. The extent of extrathyroidal extension is a key determinant of prognosis in T4a papillary thyroid cancer. Journal of surgical oncology. 2019;120(6):1016-22.

7. Sampson E, Brierley JD, Le LW, Rotstein L, Tsang RW. Clinical management and outcome of papillary and follicular (differentiated) thyroid cancer presenting with distant metastasis at diagnosis. Cancer: Interdisciplinary International Journal of the American Cancer Society. 2007;110(7):1451-6.

8. Haugen BR, Alexander EK, Bible KC, Doherty GM, Mandel SJ, Nikiforov YE, et al. 2015 American Thyroid Association management guidelines for adult patients with thyroid nodules and 
differentiated thyroid cancer: the American Thyroid Association guidelines task force on thyroid nodules and differentiated thyroid cancer. Thyroid. 2016;26(1):1-133.

9. Ford D, Giridharan S, McConkey C, Hartley A, Brammer C, Watkinson J, et al. External beam radiotherapy in the management of differentiated thyroid cancer. Clinical Oncology. 2003;15(6):33741.

10. Wang LY, Nixon IJ, Patel SG, Palmer FL, Tuttle RM, Shaha A, et al. Operative management of locally advanced, differentiated thyroid cancer. Surgery. 2016;160(3):738-46.

11. Gianoukakis AG, Giannelli SM, Salameh WA, McPhaul LW. Well differentiated follicular thyroid neoplasia: impact of molecular and technological advances on detection, monitoring and treatment. Molecular and cellular endocrinology. 2011;332(1-2):9-20.

12. Middha S, Zhang L, Nafa K, Jayakumaran G, Wong D, Kim HR, et al. Reliable pan-cancer microsatellite instability assessment by using targeted next-generation sequencing data. JCO precision oncology. 2017;1:1-17.

13. Agrawal N, Akbani R, Aksoy BA, Ally A, Arachchi H, Asa SL, et al. Integrated genomic characterization of papillary thyroid carcinoma. Cell. 2014;159(3):676-90.

14. Yoo S-K, Song YS, Lee EK, Hwang J, Kim HH, Jung G, et al. Integrative analysis of genomic and transcriptomic characteristics associated with progression of aggressive thyroid cancer. Nature communications. 2019;10(1):1-12.

15. Li B, Dewey CN. RSEM: accurate transcript quantification from RNA-Seq data with or without a reference genome. BMC bioinformatics. 2011;12(1):323.

16. Sherman BT, Lempicki RA. Systematic and integrative analysis of large gene lists using DAVID bioinformatics resources. Nature protocols. 2009;4(1):44.

17. Pozdeyev N, Gay LM, Sokol ES, Hartmaier R, Deaver KE, Davis S, et al. Genetic analysis of 779 advanced differentiated and anaplastic thyroid cancers. Clinical Cancer Research. 2018;24(13):305968.

18. Abdullah MI, Junit SM, Ng KL, Jayapalan JJ, Karikalan B, Hashim OH. Papillary thyroid cancer: genetic alterations and molecular biomarker investigations. International journal of medical sciences. 2019;16(3):450.

19. Griffith OL, Melck A, Jones SJ, Wiseman SM. Meta-analysis and meta-review of thyroid cancer gene expression profiling studies identifies important diagnostic biomarkers. Journal of Clinical Oncology. 2006;24(31):5043-51.

20. Paricharttanakul NM, Saharat K, Chokchaichamnankit D, Punyarit P, Srisomsap C, Svasti J. Unveiling a novel biomarker panel for diagnosis and classification of well-differentiated thyroid carcinomas. Oncology Reports. 2016;35(4):2286-96.

21. Schlumberger M, Lacroix L, Russo D, Filetti S, Bidart J-M. Defects in iodide metabolism in thyroid cancer and implications for the follow-up and treatment of patients. Nature Clinical Practice Endocrinology \& Metabolism. 2007;3(3):260-9. 
22. Capdevila J, Argiles G, Rodriguez-Frexinos V, Nunez I, Tabernero J. New approaches in the management of radioiodine-refractory thyroid cancer: the molecular targeted therapy era. Discovery medicine. 2010;9(45):153-62.

23. Appelbaum T, Tang W, Stopenski S, Brose M. Abstract C31: Molecular profiling in patients with radioactive iodine-refractory thyroid cancers using targeted mutation analysis. AACR; 2013.

24. Sabra M, Dominguez J, Grewal R, Larson S, Ghossein R, Tuttle R, et al. Clinical outcomes and molecular profile of differentiated thyroid cancers with radioiodine-avid distant metastases. The Journal of Clinical Endocrinology \& Metabolism. 2013;98(5):E829-E36.

25. Beroukhim R, Mermel CH, Porter D, Wei G, Raychaudhuri S, Donovan J, et al. The landscape of somatic copy-number alteration across human cancers. Nature. 2010;463(7283):899-905.

26. Kedzia C, Lacroix L, Ameur N, Ragot T, Kelly PA, Caillou B, et al. Medullary thyroid carcinoma arises in the absence of prolactin signaling. Cancer research. 2005;65(18):8497-503.

27. Peng J, Xiao L-S, Dong Z-Y, Li W-W, Wang K-Y, Wu D-H, et al. Potential predictive value of JAK2 expression for pan-cancer response to PD-1 blockade immunotherapy. TRANSLATIONAL CANCER RESEARCH. 2018;7(3):462-+.

28. Chien M-N, Yang P-S, Lee J-J, Wang T-Y, Hsu Y-C, Cheng S-P. Recurrence-associated genes in papillary thyroid cancer: An analysis of data from The Cancer Genome Atlas. Surgery. 2017;161(6):1642-50.

29. Laha D, Nilubol N, Boufraqech M. New therapies for advanced thyroid cancer. Frontiers in Endocrinology. 2020;11.

30. Kurzrock R, Ball DW, Zahurak ML, Nelkin BD, Subbiah V, Ahmed S, et al. A phase i trial of the VEGF receptor tyrosine kinase inhibitor pazopanib in combination with the MEK inhibitor trametinib in advanced solid tumors and differentiated thyroid cancers. Clinical Cancer Research. 2019;25(18):5475-84.

31. Bible KC, Suman VJ, Molina JR, Smallridge RC, Maples WJ, Menefee ME, et al. Efficacy of pazopanib in progressive, radioiodine-refractory, metastatic differentiated thyroid cancers: results of a phase 2 consortium study. The lancet oncology. 2010;11(10):962-72.

32. Cao Y, Bryan TM, Reddel RR. Increased copy number of the TERT and TERC telomerase subunit genes in cancer cells. Cancer science. 2008;99(6):1092-9.

33. Liu R, Xing M. TERT promoter mutations in thyroid cancer. Endocrine-related cancer. 2016;23(3):R143-R55.

34. Sponziello M, Silvestri G, Verrienti A, Perna A, Rosignolo F, Brunelli C, et al. A novel nonsense EIF1AX mutation identified in a thyroid nodule histologically diagnosed as oncocytic carcinoma. Endocrine. 2018;62(2):492-5.

35. Sanders Jr EM, LiVolsi VA, Brierley J, Shin J, Randolph GW. An evidence-based review of poorly differentiated thyroid cancer. World journal of surgery. 2007;31(5):934-45.

36. Vitale G, Dicitore A, Pepe D, Gentilini D, Grassi ES, Borghi MO, et al. Synergistic activity of everolimus and 5-aza-2'-deoxycytidine in medullary thyroid carcinoma cell lines. Molecular oncology. 2017;11(8):1007-22. 
37. Hanly EK, Bednarczyk RB, Tuli NY, Moscatello AL, Halicka HD, Li J, et al. mTOR inhibitors sensitize thyroid cancer cells to cytotoxic effect of vemurafenib. Oncotarget. 2015;6(37):39702.

38. Yamamoto Y, Matsui J, Matsushima T, Obaishi H, Miyazaki K, Nakamura K, et al. Lenvatinib, an angiogenesis inhibitor targeting VEGFR/FGFR, shows broad antitumor activity in human tumor xenograft models associated with microvessel density and pericyte coverage. Vascular cell. 2014;6(1):1-13.

39. Kim JW, Roh J-L, Gong G, Cho K-J, Choi S-H, Nam SY, et al. Treatment outcomes and risk factors for recurrence after definitive surgery of locally invasive well-differentiated papillary thyroid carcinoma. Thyroid. 2016;26(2):262-70.

\section{Table}

Table 3 is not available with this version.

\section{Figures}




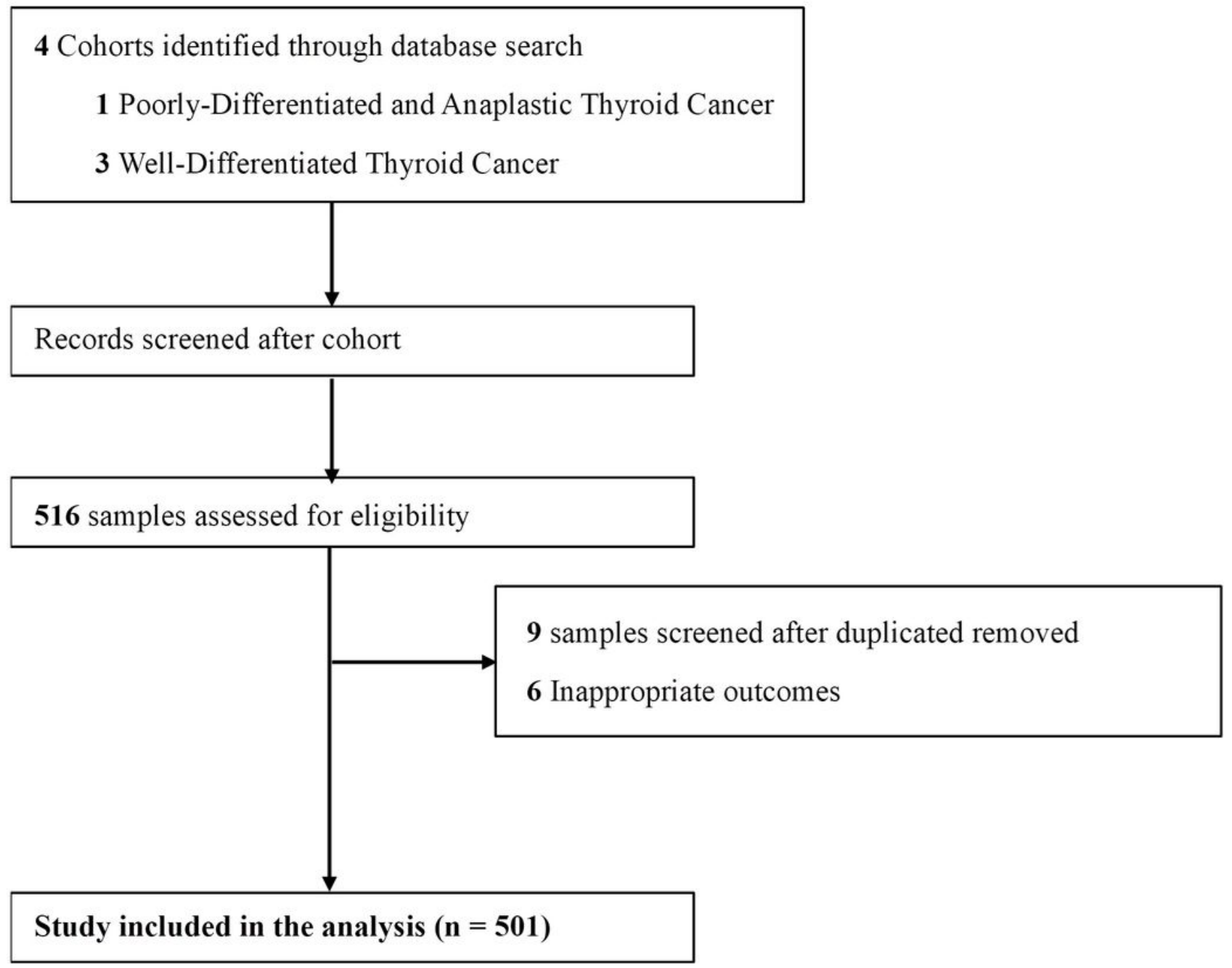

Figure 1

Flow diagram of the study selection process. 
A.

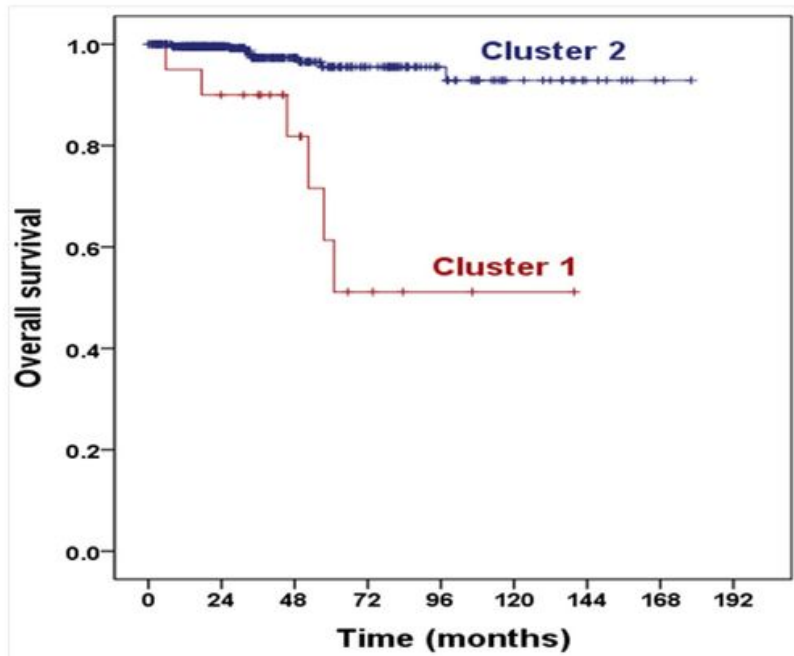

No at risk

Cluster $2478 \quad 476 \quad 471 \quad 469 \quad 468$

Cluster $123 \quad 21 \quad 20 \quad 17 \quad 17$
B.

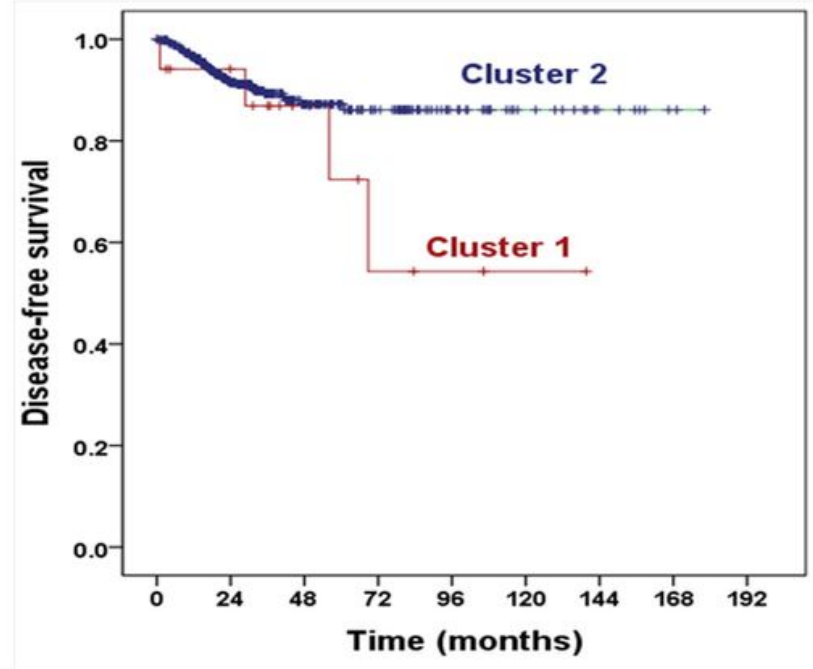

No at risk

Cluster $2469436 \quad 428427 \quad 427$

Cluster $1 \quad 18 \quad 17 \quad 16 \quad 14 \quad 14$

\section{Figure 2}

Kaplan-Meier curves estimating survival according to pathological T4 classification and T1-3 classification. A-B, Overall survival and disease-free survival between two subgroups. Log-rank test, $\mathrm{P}<.05$. 
A.
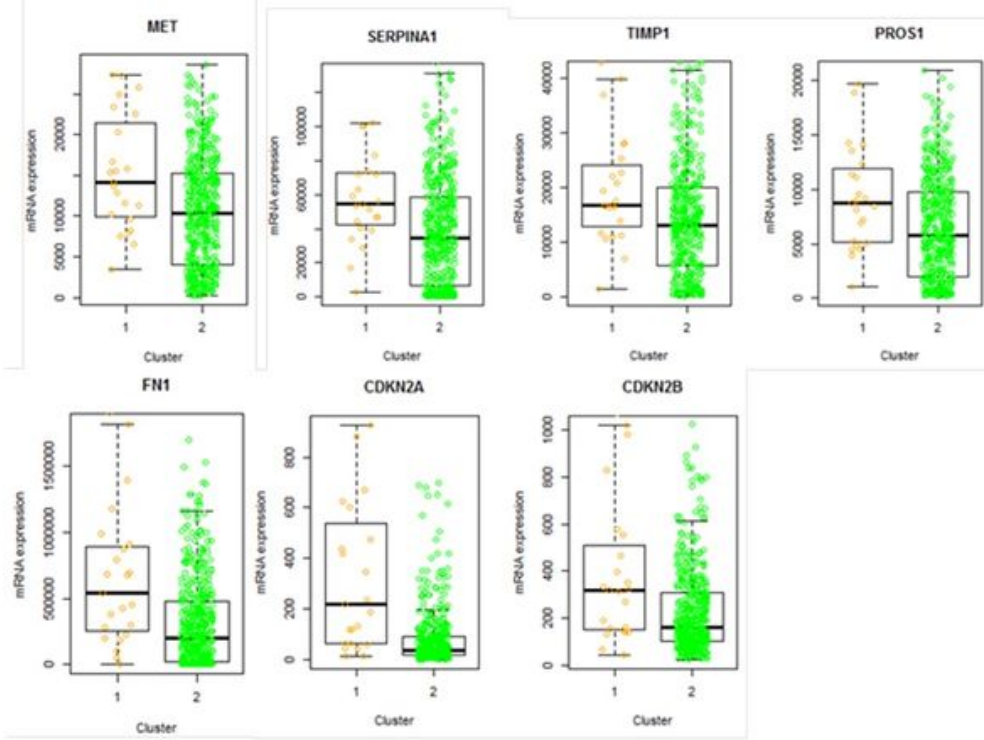

B.
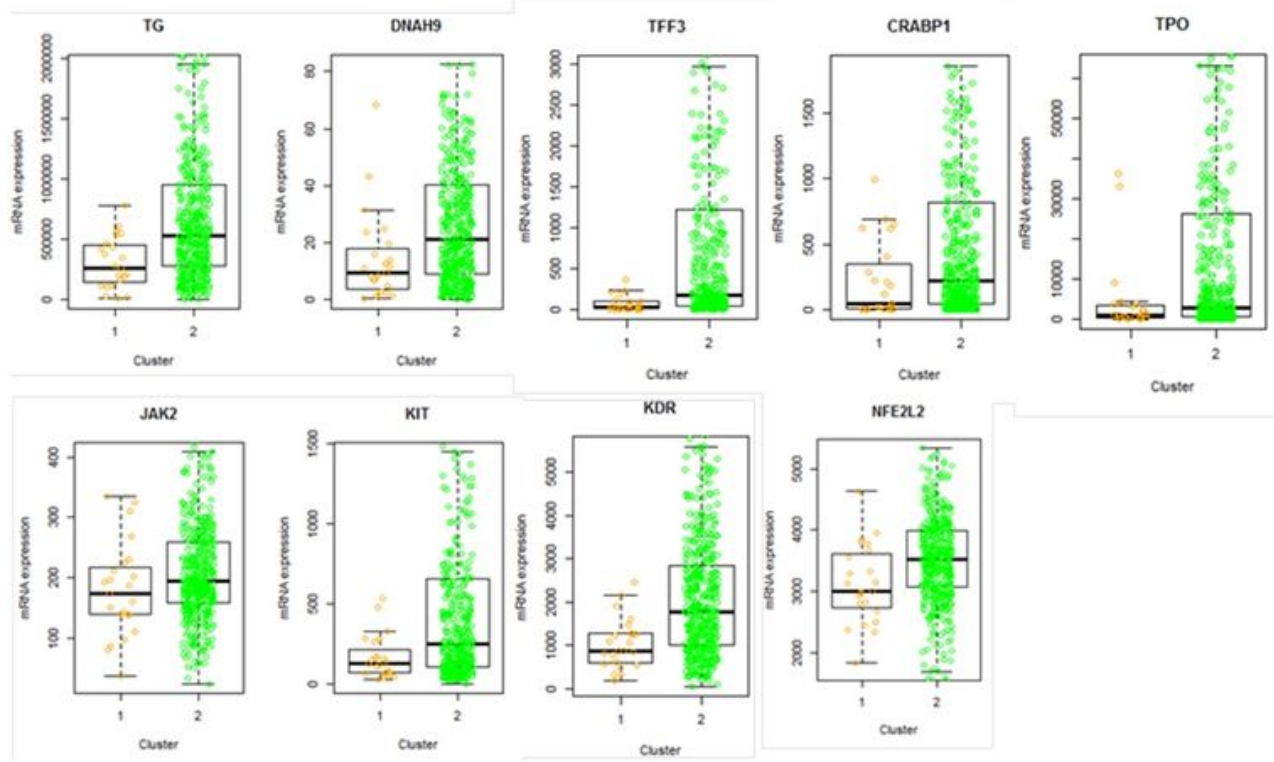

\section{Figure 3}

Identified sixteen driver genes of pathological T4 classification. A-B, The significantly gene expression elevated and lowered in Cluster1. 


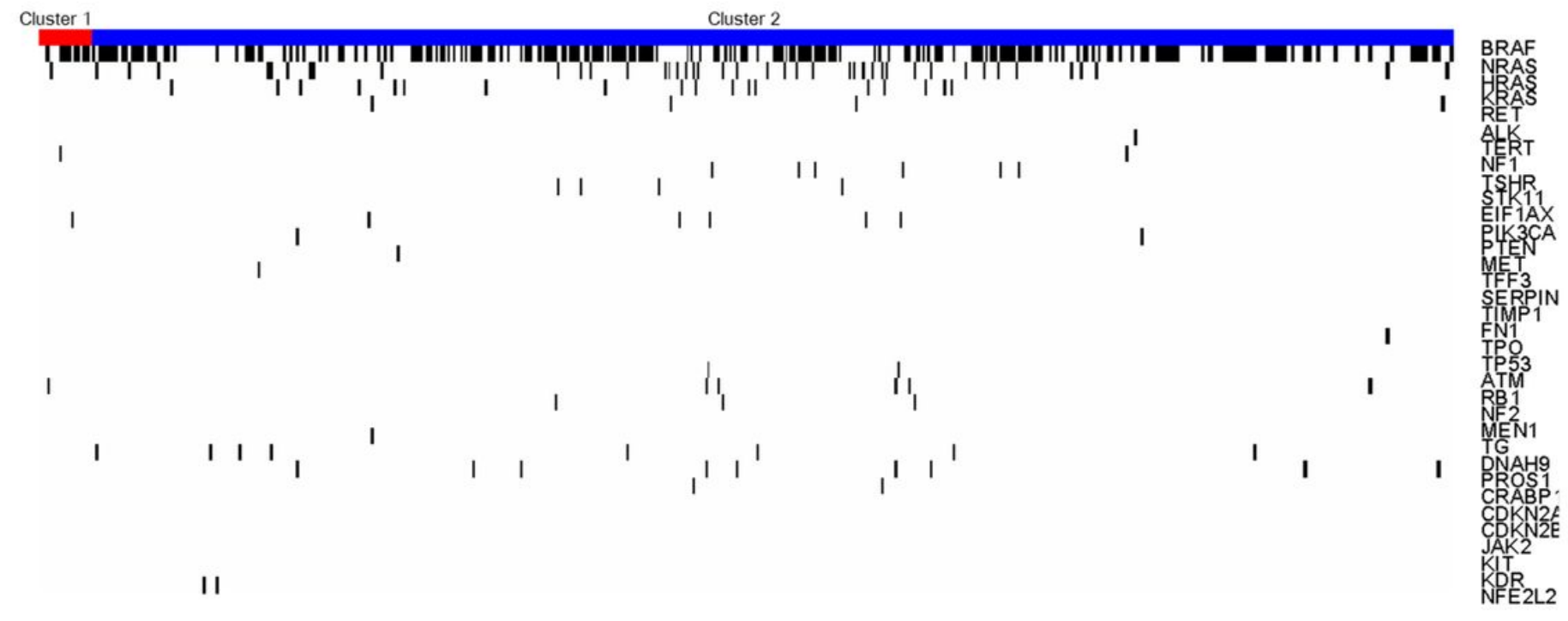

Figure 4

Somatic mutation between two clusters of patients in the TCGA cohorts. Samples are shown in columns and are cluster according to $\mathrm{T}$ classification.

\section{Supplementary Files}

This is a list of supplementary files associated with this preprint. Click to download.

- TableS1.docx 